\title{
A Bioprinted Cardiac Patch Composed of Cardiac-Specific Extracellular Matrix and Progenitor Cells for Heart Repair
}

\author{
Donald Bejleri, Benjamin W. Streeter, Aline L. Y. Nachlas, Milton E. Brown, \\ Roberto Gaetani, Karen L. Christman, and Michael E. Davis*
}

Congenital heart defects are present in 8 of 1000 newborns and palliative surgical therapy has increased survival. Despite improved outcomes, many children develop reduced cardiac function and heart failure requiring transplantation. Human cardiac progenitor cell (hCPC) therapy has potential to repair the pediatric myocardium through release of reparative factors, but therapy suffers from limited hCPC retention and functionality. Decellularized cardiac extracellular matrix hydrogel (CECM) improves heart function in animals, and human trials are ongoing. In the present study, a 3D-bioprinted patch containing CECM for delivery of pediatric hCPCs is developed. Cardiac patches are printed with bioinks composed of CECM, hCPCs, and gelatin methacrylate (GeIMA). GelMA-cECM bioinks print uniformly with a homogeneous distribution of CECM and hCPCs. hCPCs maintain $>75 \%$ viability and incorporation of CECM within patches results in a 30 -fold increase in cardiogenic gene expression of hCPCs compared to hCPCs grown in pure GelMA patches. Conditioned media from GelMA-cECM patches show increased angiogenic potential ( $>2$-fold) over GelMA alone, as seen by improved endothelial cell tube formation. Finally, patches are retained on rat hearts and show vascularization over $\mathbf{1 4} \mathrm{d}$ in vivo. This work shows the successful bioprinting and implementation of CECM-hCPC patches for potential use in repairing damaged myocardium.

\section{Introduction}

Congenital heart defects affect 35000 newborns annually, resulting in significant impairments in cardiac function and increased patient morbidity and mortality. ${ }^{[1,2]}$ Although surgical treatment methods have improved outcomes, many children end up with right-ventricular (RV) dysfunction due to increased load. ${ }^{[1,2]}$ This chronically elevated load leads to increased fibrosis and hypertrophy, resulting in RV failure. ${ }^{[1]}$ In cases where RV dysfunction persists, 18 -month survival rates are $35 \% .^{[3]}$ The

\footnotetext{
D. Bejleri, B. W. Streeter, A. L. Y. Nachlas, M. E. Brown, Prof. M. E. Davis Department of Biomedical Engineering

Georgia Institute of Technology and Emory University

1760 Haygood Dr., Atlanta, GA 30322, USA

E-mail: michael.davis@bme.gatech.edu

Dr. R. Gaetani, Prof. K. L. Christman

Department of Bioengineering and Sanford Consortium

for Regenerative Medicine

University of California, San Diego

2880 Torrey Pines Scenic Dr., La Jolla, CA 92037, USA
}

DOI: 10.1002/adhm.201800672 only restorative treatment for patients is transplantation, which is limited by the availability of donor hearts and transplant rejection. ${ }^{[2,3]}$ Even in cases where there is not critical RV dysfunction, RV output remains reduced, leading to poor quality of life.

Reparative therapies for improvement of cardiac function are critical, and although limited in pediatric populations, new treatments are being explored. ${ }^{[4-9]}$ While there have been hundreds of stem cell trials in adults, very few address pediatric populations. ${ }^{[4-6]}$ A recent study showed that intracoronary infusion of cardiosphere-derived cells can improve RV function in children and follow-up studies have been promising. ${ }^{[7]}$ In addition, bone marrow stem cells and cord blood-derived mononuclear cells also improved RV function following intramyocardial injection. ${ }^{[8]}$ We have recently shown that progenitor cells (CPCs) could improve the failing $\mathrm{RV}$ of juvenile rats subjected to pulmonary banding and a clinical trial is now underway (NCT03406884). ${ }^{[9]}$ Despite this enthusiasm, similar concerns exist in children as have been shown in adults. While CPC therapy demonstrated modest improvements in adult therapy, most CPCs were lost to circulation immediately after injection into the myocardium. ${ }^{[10,11]}$ In addition, cells are being injected into a diseased microenvironment that may not provide healthy cues for optimal CPC function. ${ }^{[11]}$

To increase retention and modify the local microenvironment, researchers have used both synthetic and natural biomaterials. ${ }^{[12,13]}$ Inclusion of appropriate cues can both direct the fate of the implanted cells, and improve the release of paracrine factors, a main mechanism of cellular therapy. ${ }^{[14,15]}$ Several studies, including ones from our laboratory, have shown that a decellularized cardiac extracellular matrix hydrogel (cECM) is a promising biomaterial used in the repair of myocardial dysfunction in adults, as well as for the delivery of stem cells. ${ }^{[16-21]}$ In prior studies, cECM increased the differentiation of rat CPCs compared to either collagen or adipose ECM alone. ${ }^{[16,20]}$ Moreover, cECM is currently in clinical trials for adults postmyocardial infarction (NCT02305602) and thus, combined with human pediatric CPCs, could rapidly advance to human testing. In adults, the material is delivered invasively through 
a catheter, which can present certain challenges. ${ }^{[19]}$ For one, the local structure of the material cannot be controlled during injection, a property that may alter stem cell phenotype. ${ }^{[22]}$ In addition, while myocardial infarction is a localized disease, heart failure due to congenital heart defects may be more global and local delivery may not be sufficient. ${ }^{[1-3]}$

One powerful method of generating controlled 3D structures for cardiac therapy is bioprinting, which has been used extensively to produce highly defined geometries of biomaterials and cells. ${ }^{[23-28]}$ Bioprinting is effective in generating polymeric scaffolds, but can be problematic for naturally derived materials. ${ }^{[24,25]}$ For the case of bioprinting ECM-derived materials, current methods rely on creating nondegradable polymeric support scaffolds, or require high concentrations of poorly printed ECM. ${ }^{[26-28]}$ The inclusion of polymers produces device-tissue and cell-material mechanical mismatch, and imposes degradation limitations. ${ }^{[29]}$ Further, finding materials that are compatible with ECM printing is not trivial. ${ }^{[30,31]}$ A bioprinting methodology that prints both cells and ECM without using nondegradable components is key in generating functional heart patches with high design control.

This work focuses on developing a bioprinted cardiac patch composed of native cECM and pediatric human CPCs (hCPCs), for use as an epicardial device that releases paracrine factors into the dysfunctional myocardium. The patch may overcome problems seen in cell therapies by retaining viable hCPCs in naturally derived cECM, and allowing for improved paracrine release from hCPCs through the bioactive cECM inducing guiding effects on cells. ${ }^{[10,16,20]}$ Additionally, the bioprinting approach allows for generation of highly defined patches with uniform component distribution. ${ }^{[23]}$ Ultimately, the patch could be used as therapy for pediatric patients suffering from $\mathrm{RV}$ failure, or perhaps even in an allogeneic manner for adult cardiac dysfunction.

\section{Results and Discussion}

\subsection{Bioprinting of hCPC/cECM Cardiac Patches}

\section{1.1. Bioprinting Acellular Structures}

Bioprinting of ECM-based materials has mainly been achieved with the inclusion of a filler polymer to allow for proper printing viscosity. ${ }^{[23,25,26]}$ ECM solutions at therapeutic concentrations $\left(6-10 \mathrm{mg} \mathrm{mL}^{-1}\right)$ are low viscosity prepolymers, which do not print effectively due to layers remaining fluid and nonoverlapping, while polymerized ECM is a fibrous material that, while more viscous then the prepolymer, comes out in "chunks" rather than a homogenous stream of print filaments. ${ }^{[18,19,26]}$ Surprisingly, cECM has been printed directly without the use of filler polymer; however, this approach suffers from two main issues. ${ }^{[26,32]}$ The first is that the required concentration for printing pure cECM $\left(20 \mathrm{mg} \mathrm{mL}^{-1}\right)$ is significantly higher than has been used in treatment studies with cECM and requires extensive harvesting from porcine sources for generation of a limited number of devices. Second, and more pressing, is that the pure cECM-printed materials are difficult to handle and risk rupture when potentially used as an epicardial patch, due to their low mechanical modulus and fibrous nature. ${ }^{[18,33]}$ In order to address this, cECM was printed with the use of filler polymers such as polycaprolactone in alternating layers, which then produced mechanical mismatch with the patch and the native myocardium, also rendering the patch with a degradation time much longer than a natural biomaterial system. ${ }^{[32]}$ Although methods have been employed to modify pure cECM mechanical properties in printed constructs, such as by inclusion of vitamin B2, it is unclear if this method can be employed as a cell-laden patch without the use of supporting polymer layers. ${ }^{[27,32]}$

To generate a cECM patch that has a high degree of printability, proper mechanical properties for myocardial therapy, and allows for cell viability and paracrine release, we used gelatin methacrylate (GelMA) as a support material. GelMA is a natural biomaterial based on collagen, which has methacrylate groups grafted onto the gelatin structure so that the material can undergo radical polymerization. ${ }^{[34,35]}$ GelMA is used extensively as a bioactive and resorbable material for regenerative medicine applications, and in a multitude of tissues such as muscle, liver, and bone. ${ }^{[34-36]}$ In order to limit cell damage, we employed a white light system for gel polymerization after structure formation. This white light system has advantages over UV systems that otherwise induce increased cell death and stress. ${ }^{[37-39]}$ We investigated the use of various cross-linking systems, such as ruthenium-sodium persulfate or Irgacure 2959, but found that an Eosin Y system allowed for the most effective formation of structurally reliant and viable patches. ${ }^{[35,38,39]}$ Most importantly for bioprinting, GelMA undergoes a polymerization when cooled from physiological temperatures to below $10^{\circ} \mathrm{C}$, and is viscous even at room temperature with concentrations of $10 \%$ weight/volume (w/v) and above. This phase transition makes it suitable for bioprinting as a natural material, and has been used often for this application. ${ }^{[39]}$ This work utilized $5 \% \mathrm{w} / \mathrm{v}$ GelMA in the bioink formulations so that the bioink was still a significant portion $\mathrm{CECM}\left(8 \mathrm{mg} \mathrm{mL} \mathrm{L}^{-1}\right)$, compared to increasing the concentration of GelMA to $10 \%$ or higher, which would have produced a bioink that is mostly GelMA with some cECM added. In addition, low w/v \% GelMA supports more effective cellular outcomes such as viability and proliferation. ${ }^{[40]}$ Our printing strategy involved cooling $5 \% \mathrm{w} / \mathrm{v}$ GelMA to $10{ }^{\circ} \mathrm{C}$ for $10 \mathrm{~min}$ to allow for gelation and enhanced printing viscosity of the cECM/hCPC bioink. An overview of the printing strategy is seen in Figure 1.

The printing methodology allowed for clean and defined extruded filaments when printing either GelMA or GelMAcECM (Figure 2A). To ensure that the cECM fibers were uniformly distributed in the printed structures, we stained the cECM with AF568, which forms a strong bond to primary amines on the CECM proteins. The red staining in Figure 2B is the $\mathrm{CECM}$ fibers, indicating that the $\mathrm{CECM}$ was distributed homogeneously throughout the entire printed structure, rather than in clumped locations such as filament junctions. A higher magnification in 3D of a printed filament in Figure 2C shows that the cECM formed homogeneously distributed dense fibers after polymerization at physiological $\mathrm{pH}$ and temperature. We quantified the printability of the structures using a parameter based on the extent to which the holes between filaments 


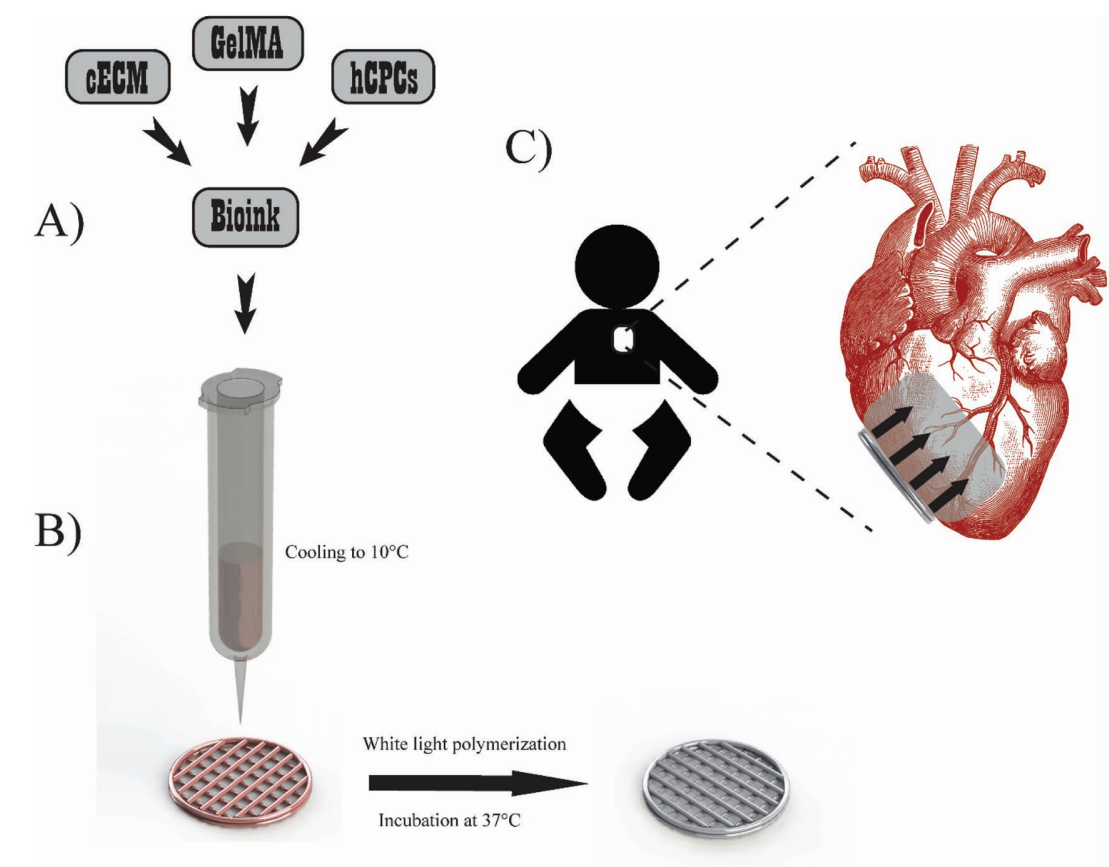

Figure 1. Printing overview. A) Bioink preparation involved combining $C E C M, h C P C s$, and GelMA to form naturally derived and cell-laden materials for printing. B) Printing methodology involved cooling the bioink to $10{ }^{\circ} \mathrm{C}$ in the $3 \mathrm{D}$ bioprinter barrels to allow GelMA polymerization for improved printability. Patches were printed with infill patterns of $90^{\circ}$ intersecting filaments and contour. Patches were polymerized via white light to induce radical polymerization of GelMA, followed by incubation at $37^{\circ} \mathrm{C}$ for at least $1 \mathrm{~h}$ to induce $\mathrm{CECM}$ polymerization. C) Patch implementation will involve pericardially inserting the patch to the RV of pediatric patients, where the patch will release key proregenerative paracrine factors.

match a square shape, as previously described and discussed in the Experimental Section. ${ }^{[41]}$ A value of printability close to 1.0 demonstrates ideal gelation, and thus the printing property, of the bioink. This value shows that the holes are close to a perfect square shape due to the filaments being uniform in thickness, homogeneous, and rigidly defined with multiple layers stacking on one another. As can be seen in Figure 2D, both GelMA and GelMA-cECM bioinks had printability close to a value of 1.0 and the inclusion of cECM improved the printability of the bioink significantly to achieve a value closest to ideal printing.

\subsubsection{Bioprinting hCPC-Laden Structures and Cardiac Patches}

Following incorporation of $\mathrm{CECM}$ in to the printed structure, we next sought to determine if primary cells could be added to the printing mix. To perform this, hCPCs were incorporated into the bioinks and evaluated for effectiveness in creating homogeneously distributed cell-laden print structures. Nonextrusion-based bioprinting methods require high printing pressures that render cells nonviable or methodologies that result in dispersion of cells toward the edges of printed constructs, rather than homogeneously distributed throughout. ${ }^{[42,43]}$ In addition, cells can leach out of printed hydrogel constructs if the materials are soft and not effectively polymerized, resulting in a loose network. ${ }^{[4]}$ As shown in the bright field images in Figure 3, we were able to add cells to the print for both GelMA alone (Figure 3A) and GelMAcECM (Figure 3B), where cells were retained in the gels after cross-linking. To obtain a clearer image of hCPCs throughout the test grids, cells were stained with a lipophilic dye (DiD) prior to printing. Figure 3C shows the printed grids after swelling, indicating that the cells appeared homogeneously distributed throughout the filaments. To quantify distribution, an averaged fluorescence line scan along filaments showed that the fluorescence intensity throughout the filaments was uniform and that the cells were homogeneously distributed (Figure 3D). Cells were incorporated throughout the filaments, and GelMA-cECM grids once again appeared to have better printability, as indicated by the hole geometry, where the GelMA-cECM grids had more square holes than GelMA grids. Printing parameters were not modified by the incorporation of cells, maintaining a low printing pressure (0.7-0.8 bar), and thus low shear stress, on the cells. In addition, cells remained firmly supported within the printed constructs, with no cells leaching out of the grids or sifting to the bottom of the filaments.

The hCPC/cECM bioink was shown to have ideal printability with homogeneous distribution of both cECM and hCPCs throughout the printed structures, as described in the above sections. Moving on, we were able to create cardiac patches using the cell-laden bioink, based on a cylindrical shape, as indicated in Figure 4. Figure 4A shows the printed patches prior to white light polymerization while Figure $4 \mathrm{~B}$ shows the CAD models using the patch design. The patches were pink due to the Eosin Y photoinitiator and change to clear after polymerization. The printed patches maintained the same shape and structure as the CAD model, due to the high printability bioink. The infill pattern of the patches were perpendicular aligned filaments generated through multiple print layers, indicating further degrees of printing control and structure fidelity.

\section{2. hCPC Viability, Differentiation, and Proliferation within Bioprinted Cardiac Patches}

Evaluating the viability of cells within the cardiac patches is critical to ensure live cells that can participate in producing important proreparative paracrine factors. ${ }^{[12,15]}$ Evaluation of cell viability directly is also critical within bioprinted scaffolds, particularly because bioprinting has been shown to reduce cell viability in printed constructs due to high shear stresses on the cells from small diameter needle tips, such as the tips used in this study. ${ }^{[42,43]}$ In addition, cells grown in thick 3D structures can suffer death due to lack of nutrient diffusion, particularly at the center of the structures, producing a necrotic core. ${ }^{[42,45]}$ As shown in Figure 5, hCPCs within printed cardiac patches 

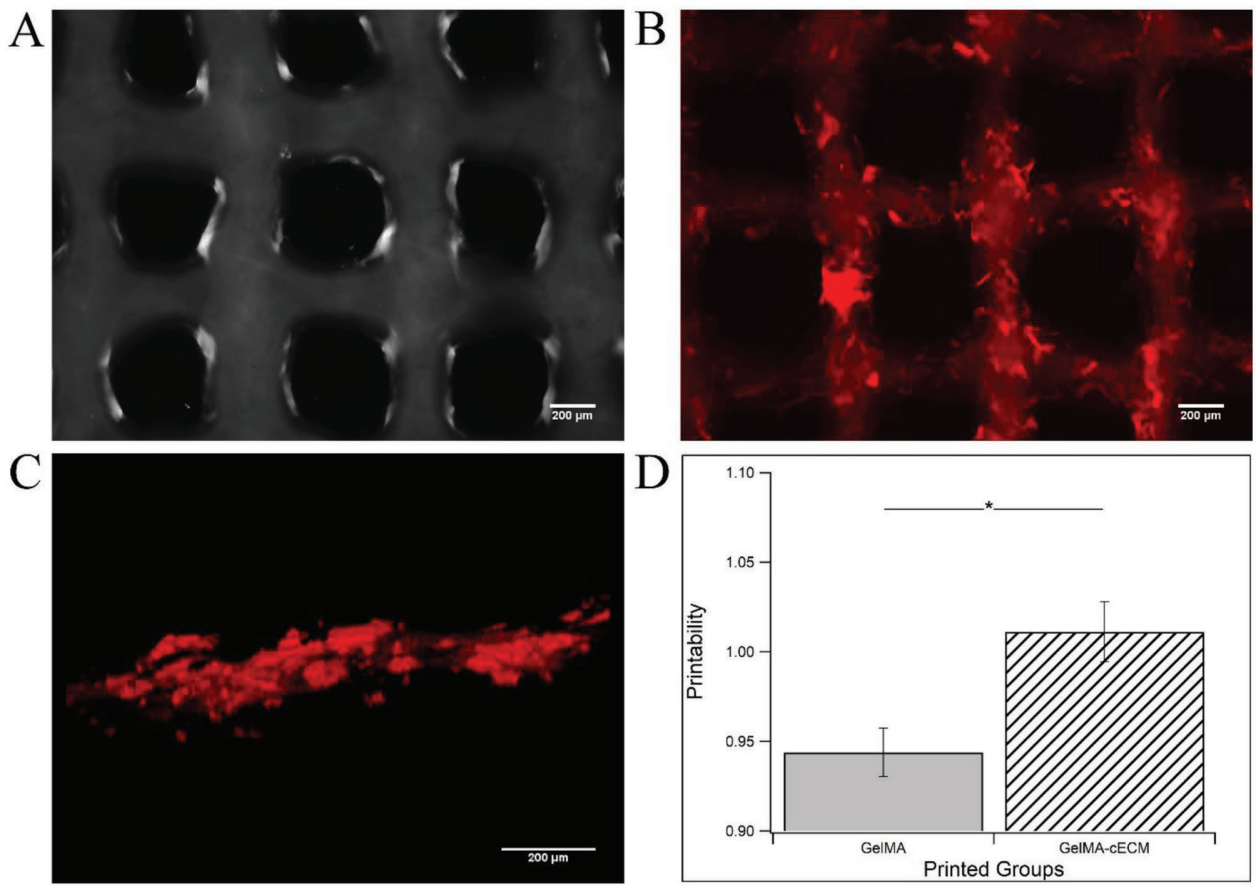

Figure 2. Printability analysis of GelMA-cECM bioinks. A) Bright-field image of printed test grids of GelMA. B) Fluorescence image of printed test grids of GelMA-cECM with staining for cECM by AF568. C) 3D fluorescence close-up view of printed filament of GelMA-cECM, with staining for cECM by AF568. D) Printability comparison between GelMA and GelMA-cECM bioinks. $*=p$-value $<0.03$, given by paired $t$-test, $n=5$.

were stained to determine the total number of dead (red) to live (green) cells for either GelMA (Figure 5A) or GelMA-cECM (Figure 5B). Cell viability was quantified by measuring the number of live and dead cells at different locations and heights within the cardiac patch at days 1, 3, and 6 after formation and showed high viability, from 70 to $80 \%$ live cells on average
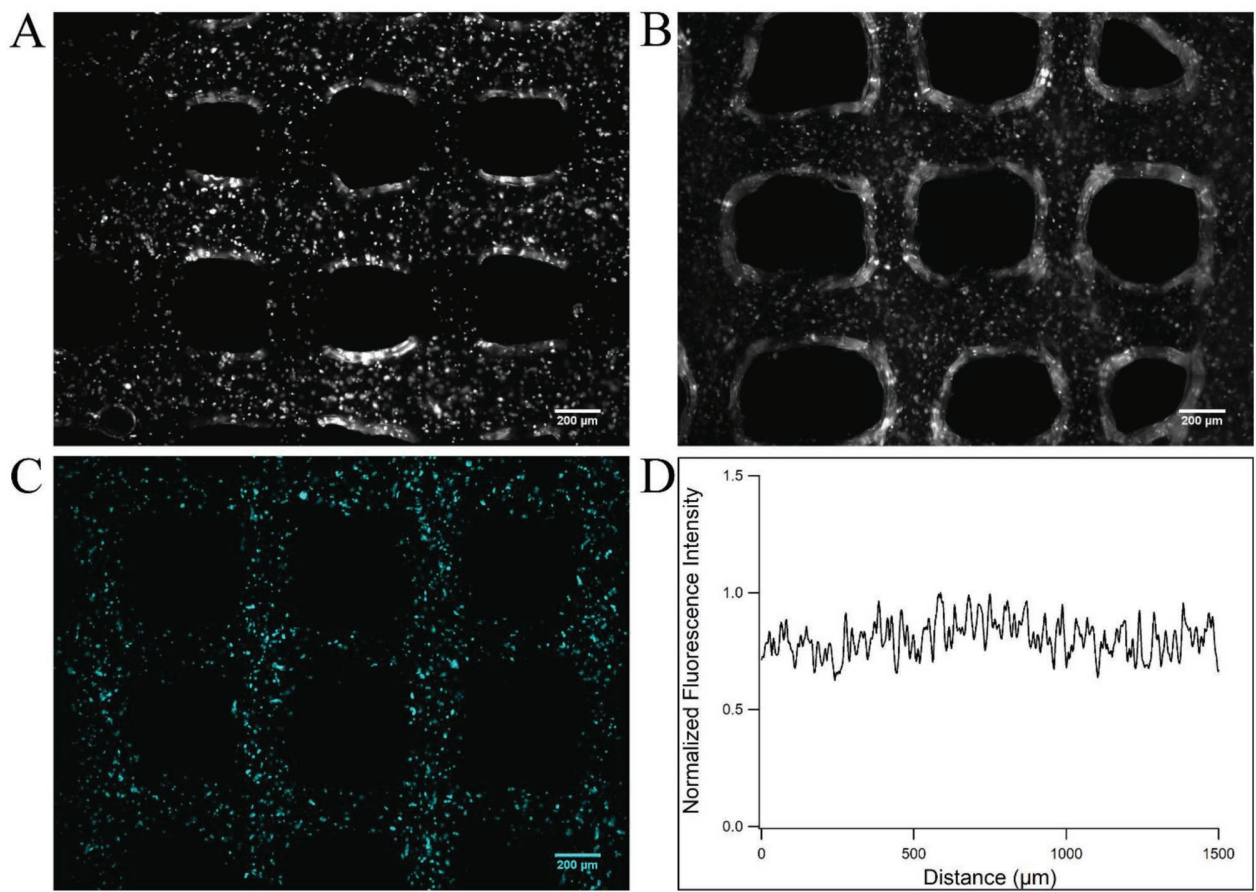

Figure 3. Printing hCPC-containing bioinks. A) Bright-field image of printed test grids of GelMA bioinks containing hCPCs, taken $1 \mathrm{~h}$ after printing. B) Brightfield image of printed test grids of GelMA-cECM bioinks containing hCPCs. C) Fluorescence image of printed test grids of GelMA-cECM with hCPCs stained with DiD. D) Normalized fluorescence intensity of line scans performed on stained hCPC test grids. Line scans were performed across several filaments. 

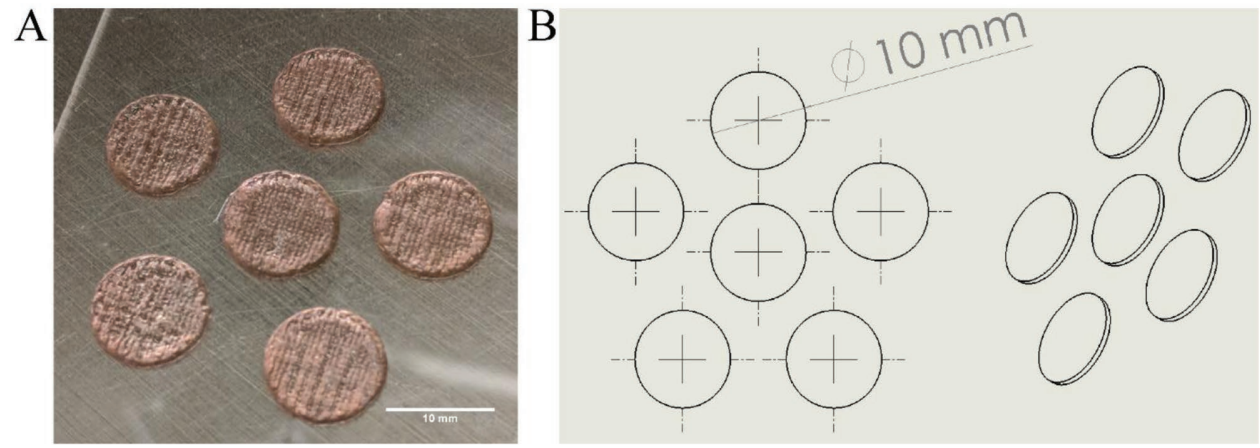

Figure 4. Printed patches. A) Printed patches of $10 \mathrm{~mm}$ diameter and $0.6 \mathrm{~mm}$ height. Patches are printed uniformly from patch to patch, and the grid infill pattern can be seen. Patches are pink postprinting due to inclusion of photoinitiator Eosin $\mathrm{Y}$, and become clear postpolymerization. B) CAD model sketch used for patch printing.
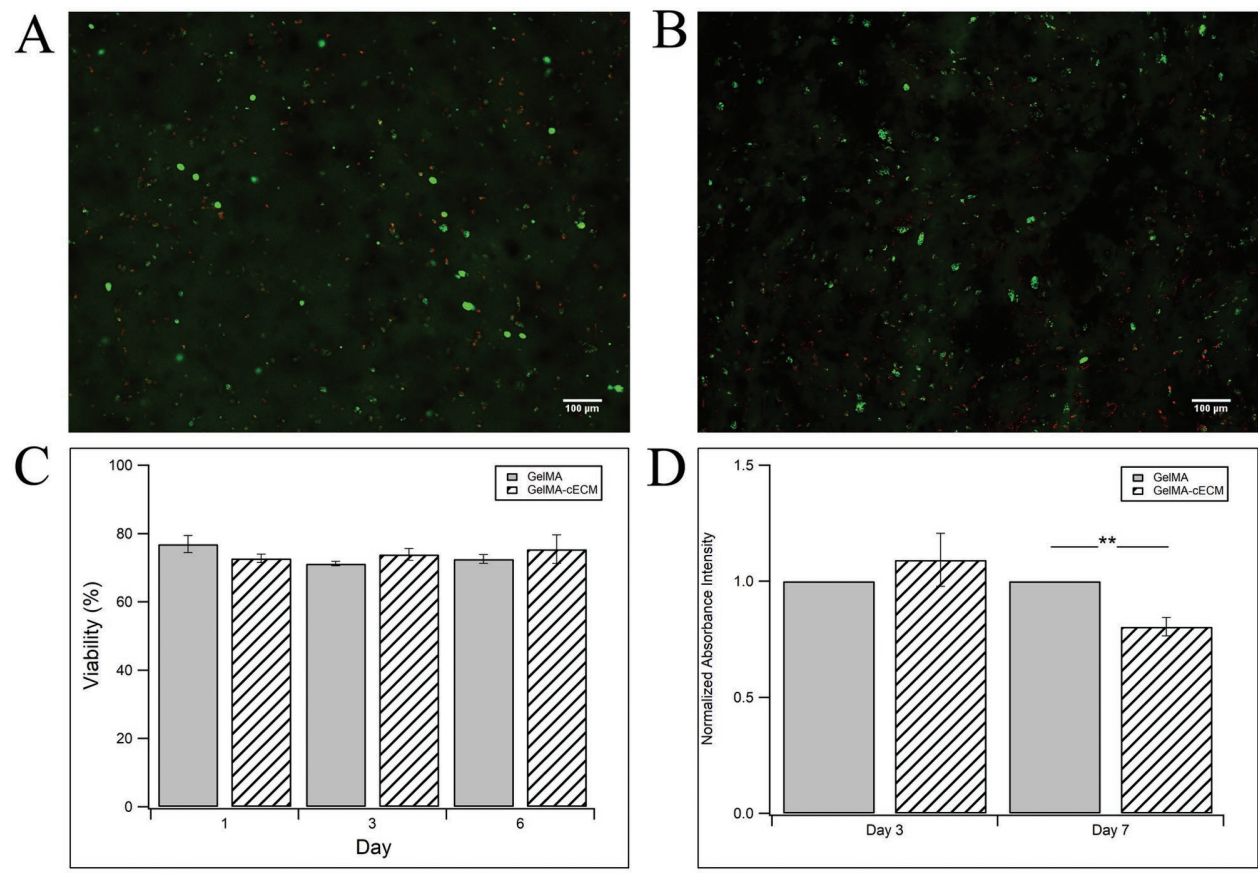

$\mathrm{E}$

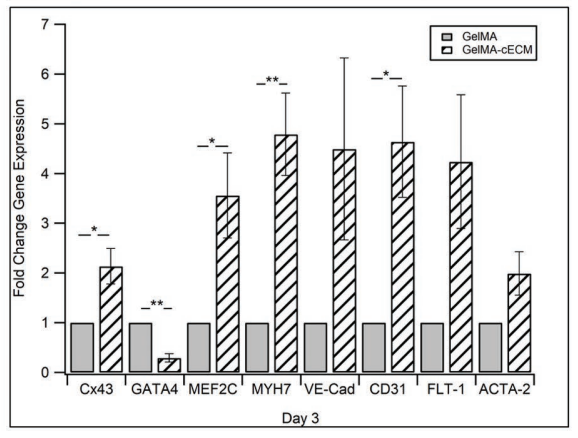

$\mathrm{F}$

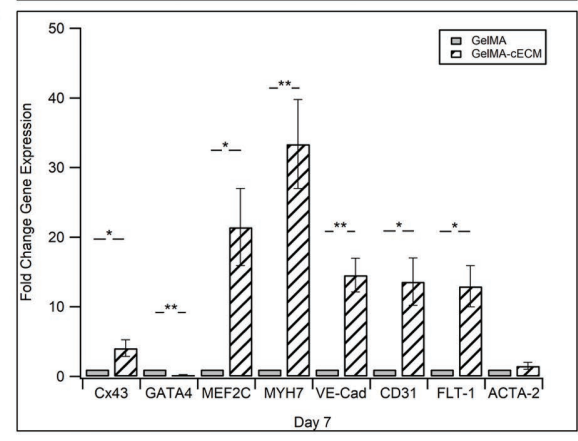

Figure 5. hCPC functionality within printed patches. A) Characteristic live/dead fluorescence image of hCPCs in GelMA patches, with live cells marked green (Calcien AM) and dead cells marked red (EtD) at $1 \mathrm{~d}$ after formation. B) Characteristic live/dead fluorescence image of hCPCs in GelMA-cECM patches at $1 \mathrm{~d}$ after formation. C) Viability of hCPCs in printed patches at 1, 3, and $6 \mathrm{~d}$. D) Proliferation of hCPCs in printed patches at 3 and $7 \mathrm{~d}$, where absorbance intensity is normalized to the measured absorbance of hCPCs in GelMA patches in all experiments. E) Fold change gene expression over hCPCs in GelMA patches for Cx43, GATA4, MEF2C, MYH7, VE-Cad, CD31, FLT-1, and ACTA-2 at day 3. F) Fold change gene expression over hCPCs in GelMA patches for Cx43, GATA4, MEF2C, MYH7, VE-Cad, CD31, FLT-1, and ACTA-2 at day 7. * $=p$-value $<0.05$, ** $=p$-value $<0.005$, given by ANOVA with Tukey's post-test, $n=3-6$ for all samples at all time points. 
seen in Figure 5C. There was no significant difference between groups or time points when comparing the percent of viable cells. Throughout all structures, there was no necrotic core or reduction of cell viability, indicating that nutrient diffusion was likely not a factor. The cell viability overall was most likely not impacted significantly by the printing methodology, or if there were effects to the cells due to the printing, the degree of cell damage was mitigated by the material being an effective environment for cell growth and nutrient diffusion coupled with printing of aligned fibers which may be beneficial to cell function.

Proliferation and differentiation are additional parameters that are important in characterizing functionality of hCPCs in bioprinted patches. hCPC-laden patches were grown in culture media supplemented with EdU for 3 and 7 d, and absorbance intensity from GelMA-cECM patches was normalized to values measured from GelMA patches. While there was no difference between the proliferation of hCPCs in GelMA and GelMA-cECM patches after $3 \mathrm{~d}$, as seen in Figure 5D, hCPCs in GelMA-cECM patches had reduced proliferation compared to GelMA patches after $7 \mathrm{~d}$. Similarly, hCPC-laden patches were grown for 3 and $7 \mathrm{~d}$, and the fold change in genetic expression of key cardiac, endothelial, and smooth muscle genes from hCPCs in GelMA-cECM patches compared to hCPCs in GelMA patches was assessed through polymerase chain reaction (PCR). Analysis of gene expression of cardiac transcription factors GATA4 and myocyte enhancement factor 2C (MEF2C) and cardiac-specific proteins connexin $43(\mathrm{Cx} 43)$ and $\beta$-myosin heavy chain (MYH7), endothelial cell markers vascular endothelial cadherin (VE-Cad), platelet endothelial cell adhesion molecule (CD31), and vascular endothelial growth factor receptor 1 (FLT-1), and smooth muscle marker $\alpha$-smooth muscle actin (ACTA-2) was evaluated at 3 and 7 days, as seen in Figure 5E,F. hCPCs in GelMA-cECM patches showed enhanced cardiac differentiation through increased expression of MEF2C, Cx43, and MYH7, and decreased expression of GATA4, an early differentiation marker, indicating that the hCPCs in GelMA-cECM patches were moving toward later differentiation than hCPCs in GelMA patches. hCPCs in GelMA-cECM patches also showed increased expression of endothelial marker CD31 at day 3, although there was no difference in expression of endothelial markers VE-Cad and FLT-1 or smooth muscle marker ACTA-2. At day 7, expression of all cardiac and endothelial markers was increased in GelMA-cECM patches, with higher fold-change values than day 3. ACTA-2 remained unchanged between groups at both days. Thus, we conclude that incorporation of cECM into patches improved both cardiac and endothelial differentiation of hCPCs, while not influencing smooth muscle differentiation. The enhanced differentiation of hCPCs in cECM incorporated patches at day 7 mirrors the proliferation trends seen in Figure 5D, as stem cells most often show reduced proliferation with increased committed. These assessments also reaffirm the results measured for hCPCs in 2D culture, where cECM improved differentiation of CPCs compared to cells grown on collagen-based materials. ${ }^{[16]}$ Regardless, it is clear that while hCPCs remained viable in printed patches, the inclusion of cECM improved differentiation and reduced proliferation of hCPCs, which in turn may improve paracrine potential of hCPC-laden GelMA-cECM patches.

\subsection{Proangiogenic Potential of hCPC/cECM Cardiac Patches}

Many studies now attribute the true benefit of cell therapy to be release of paracrine factors. ${ }^{[15,46-50]}$ To evaluate paracrine release, we grew cell-laden patches in treatment media for up to $7 \mathrm{~d}$ and collected the conditioned media every $2 \mathrm{~d}$. We then performed a tube formation assay using human umbilical vein endothelial cells (HUVECs) cultured on Matrigel with conditioned media. HUVECs grown in either nonconditioned treatment media or endothelial cell growth media with supplemented growth factors showed similar values for total tube length formed, so nonconditioned treatment media was used as positive controls. As shown in Figure 6, HUVECs formed tube-like structures when cultured in conditioned media taken from both cell-laden GelMA (Figure 6A) and GelMA-cECM (Figure 6B) patches. When comparing the angiogenic potential of cell-free patches, seen in Figure 6C, there was no difference between GelMA and GelMA-cECM groups. In contrast, the angiogenic potential of media collected from cell-laden GelMAcECM patches was significantly higher than media from GelMA patches alone at day 3, while both groups showed improved angiogenic potential at day 7 compared to day 3. While GelMAcECM was superior at both time points, both groups showed an increase in angiogenic potential over time. While there are many other parameters that conditioned media may alter, angiogenesis may be one of the most important for improving cardiac function. Additionally, we may be underestimating the effects as some growth factors released may interact with the GelMA and/or cECM and prevent release into the conditioned media, as studies have shown that growth factors, such as heparin binding growth factor and hepatocyte growth factor, bind to $\mathrm{CECM}$ and are released gradually. ${ }^{[51,52]}$

In developing effective hydrogels for soluble factor release, it is key to generate materials that are stiff enough to allow for scaffold stability, but do not have too dense a network that limits cellular functions and release of signaling factors. ${ }^{[44]}$ Systems that balance these parameters have been developed around natural or synthetic materials, many of which prove effective in releasing reparative factors into damaged tissues, whether through encapsulation of regenerative cells or the factors themselves. ${ }^{[46-49]}$ The hCPC-laden GelMA-cECM patches developed here also allowed for generation of a solid patch with enhanced factor release. These results are similar to other materials that have been developed, but with incorporation of cardiac specific cells and matrix that may release cardiac-specific paracrine factors. This cardiac specificity in the patch design may be most beneficial in repairing the damaged myocardium, as opposed to using non-tissue-specific biomaterials such as GelMA. ${ }^{[19,46,50]}$

\subsection{Mechanical Characterization of Bioprinted Patches}

Mechanical properties of biomaterials play a critical role in modulating cellular function. Stem cells are more viable, proliferative, and produce more effective regenerative outcomes when grown in hydrogels that match the properties of native tissue. ${ }^{[12,53]}$ This is also true as well for hCPCs, where cells perform more effectively when grown in materials that match the mechanical modulus of native myocardium from 5 to $15 \mathrm{kPa} .{ }^{[54]}$ 

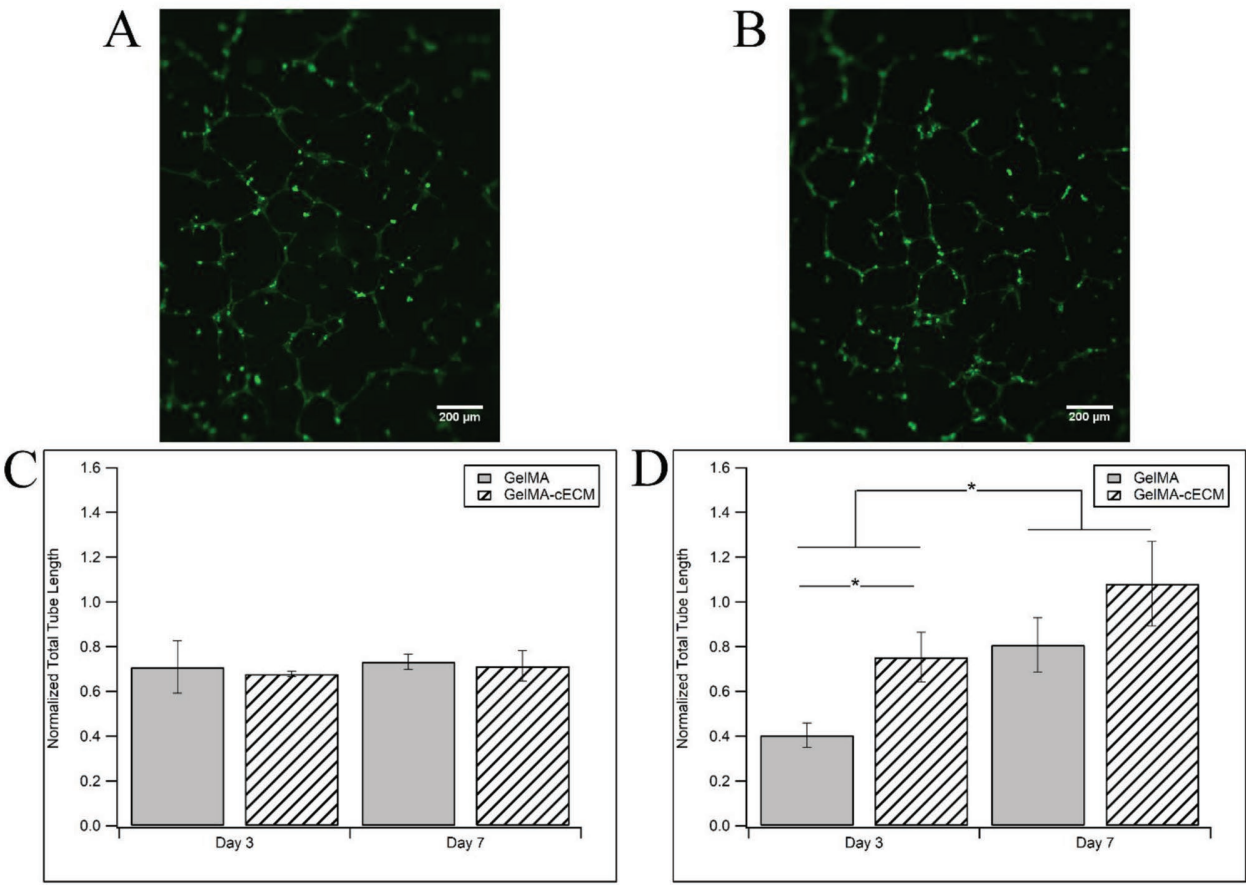

Figure 6. Angiogenic potential of cardiac patches. A,B) Characteristic HUVEC tube formation after $6 \mathrm{~h}$ when grown with conditioned media collected at day 7 from cell-laden GelMA and GelMA-cECM patches. C,D) Total HUVEC tube length normalized to positive controls for cell-free and cell-laden patches. $*=p$-value $<0.05$, given by ANOVA with Tukey's post-test, $n=3-6$ for all samples at all time points.

A material modulus that more closely matches the myocardium also ensures there is limited mechanical mismatch between the hydrogel and the heart, which can otherwise cause problems such as dissection, buckling, or immune responses. ${ }^{[29,55]}$ While GelMA and GelMA-cECM patches are evaluated in this study, we also evaluated the use of modifying the mechanical properties of the patches by adding acrylate groups using $\mathrm{N}$-succinimidyl acrylate, as employed in previous studies. ${ }^{[33]}$ While the modification increased stiffness over GelMA and GelMA-cECM groups, the patches more readily degraded compared to both groups and did not alter the hCPC viability or paracrine function over GelMA-cECM (data not shown), so this direction was not pursued further, although the properties of the patch could potentially be modified through this method.

As seen in Figure 7A, the modulus of pure GelMA was $3000 \mathrm{~Pa}$, similar to published studies, though short of the native myocardium. ${ }^{[56]}$ Incorporation of cECM significantly increased the modulus to $5000 \mathrm{~Pa}$, indicating that the material properties of the GelMA-cECM patch could be tailored within physiological ranges. In addition to stiffness, we also measured swelling ratio in Figure 7B. All samples were sufficiently hydrated, with a swelling ratio between 9 and 12. There was a decrease in the swelling ratio between the GelMA and GelMAcECM groups, which was expected as increases in stiffness suggest a tighter polymer network and result in more liquid exclusion.

As a cardiac patch must persist for the repair process, degradation time is a critical parameter. We evaluated the degradation of patches and materials in cell treatment media over $21 \mathrm{~d}$ by examining both the change in wet weight (Figure 7C) and change in stiffness (Figure 7D). When comparing weight change of printed patches or the change in stiffness of the materials, both groups remained solid and did not significantly degrade over the range of $21 \mathrm{~d}$. Although the data presented describe the degradation of patches without cells, patches that incorporated cells lasted the duration of testing shown in Sections 2.3 and 2.4 with no observable degradation. It is important to describe this method of degradation as only evaluating hydrolysis, as opposed to exposing the patch to conditions seen in vivo including proteases, cyclic strain, and shear stresses. To evaluate the degradation of the patches in a more physiological relevant environment, hCPC-laden materials were cultured in conditioned media harvested from cardiac fibroblasts (cFBs), which would be present in cases of ventricular remodeling and hypertrophy. The cFB conditioned media more closely captures the environment of patches in vivo due to incorporation of a complex mixture of remodeling components, compared to commonly employed incubation in collagenase I, which degrades the patches in a matter of hours and may not be as physiologically relevant. As seen in Figure 7E, hCPC-laden GelMA materials did not degrade or change mechanical modulus over the course of 7 and $21 \mathrm{~d}$ in $\mathrm{CFB}$ conditioned media. Interestingly, while hCPC-laden GelMA-cECM materials did not degrade or change mechanical modulus over the course of $7 \mathrm{~d}$, by $21 \mathrm{~d}$ the material stiffness increased compared to the stiffness at both 1 and $7 \mathrm{~d}$. The change in stiffness at $21 \mathrm{~d}$ is also significantly higher than hCPC-laden GelMA materials at the same time and may be due to stimulation of the hCPCs to remodel their environment. Regardless, the GelMA-cECM patches, both with and without hCPCs, do not degrade in vitro over an extended timeframe and may be suitable for extended retention in vivo. 

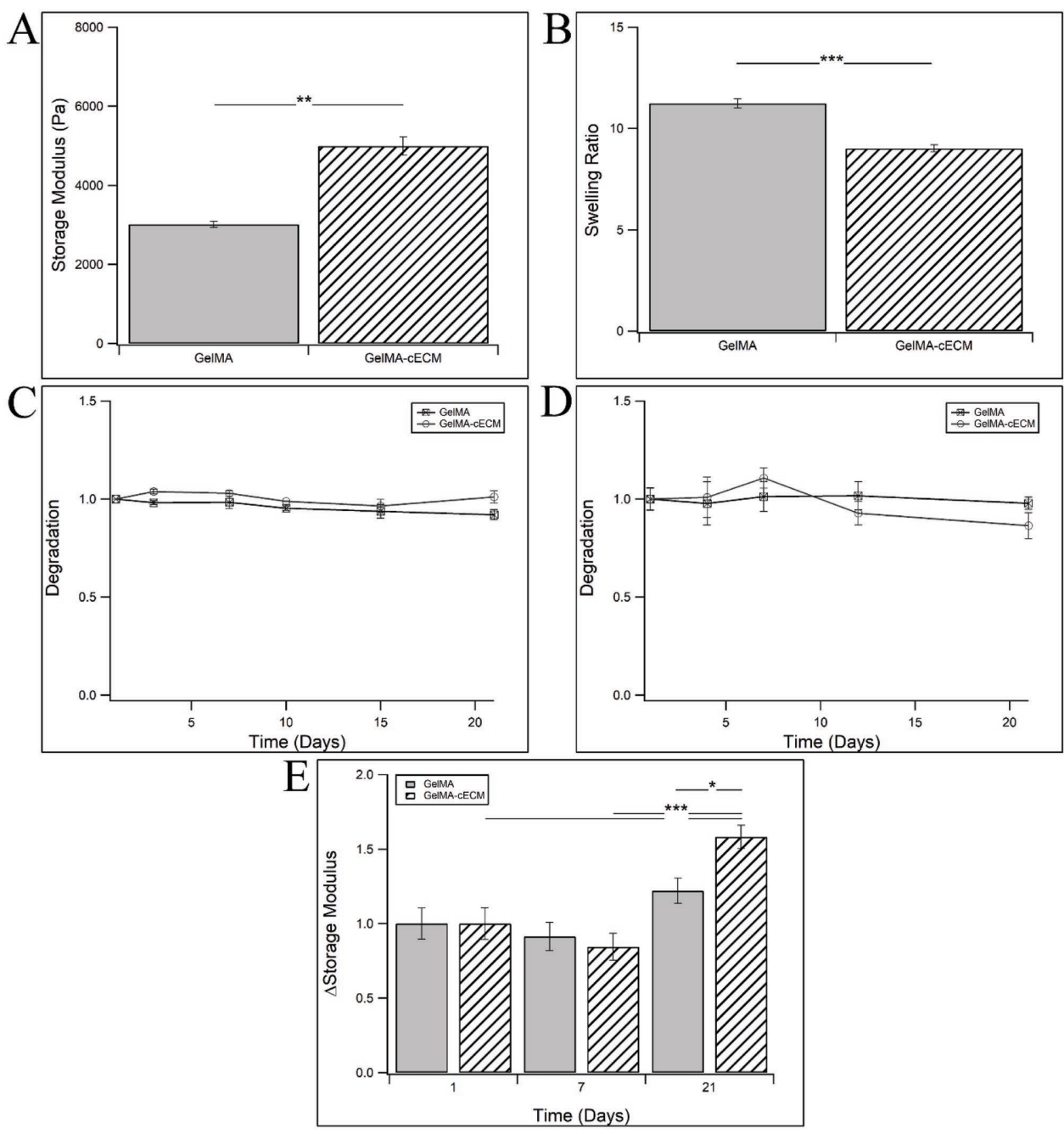

Figure 7. Material analysis of printed patches. A) Viscoelastic storage moduli of GelMA and GelMA-cECM. B) Swelling ratio of GelMA and GelMACECM patches. C) Degradation of patches, measured as the sample weight compared to initial weight of the patches postswelling. D) Degradation of cell-free materials in cell culture media, measured as the sample storage modulus compared to the initial modulus of the material postswelling. E) Remodeling of hCPC-laden materials grown in CFB conditioned media, measured as the sample storage modulus compared to the initial modulus of the material postswelling. $*=p$-value $<0.05$, $* *=p$-value $<0.01$, $* * *=p$-value $<0.005$, given by ANOVA with Tukey's post-test, $n=3$ for all samples in all subfigures.

\subsection{In Vivo Implantation of GelMA-cECM Patches}

Attachment of GelMA-cECM patches onto the epicardial surface is critical to ensure the devices can be deployed with minimal manipulation. We evaluated the potential of the patches to remain attached to rat hearts after placement on the epicardium. As can be seen in Videos S1 and S2 (Supporting Information), surgical attachment of the patches was achieved on rat hearts via placement on top of the epicardial surface of the right ventricle after opening the chest cavity and exposing the beating heart. Three methods of attachment were evaluatedsimple placement on the epicardium without secondary support, placement on the epicardium followed by covering with the pericardium, and placement on the epicardium with a single suture. All three methods allowed for patch placement on beating rat hearts, without buckling or patch damage. Prior to patch generation, cECM was incubated with a fluorescent dye for postimplantation imaging. The simple placement without secondary support resulted in patch movement, indicating that some sort of support was needed for patch retention. Following the pericardial and suturing methods, hearts were excised from rats and fluorescently imaged to determine if the patch remained on the heart. Of eight patches implanted, all were retained up to 7 and $14 \mathrm{~d}$, regardless of pericardial or suturing attachment method. As seen in Figure 8, patches were retained at day 7 (Figure 8A suture), and day 14 (Figure 8B pericardial, Figure $8 \mathrm{C}$ suture) with clear and stable fluorescent signal up to $14 \mathrm{~d}$. Both methods did not require the use of surgical adhesive such as fibrin, which may impose a barrier layer for paracrine release. ${ }^{[57]}$ However, the pericardial method required cutting of the patches into smaller than $10 \mathrm{~mm}$ sizes before placement in two out of the three animals evaluated with this method, as the full patch tended to fold upon covering with the pericardium, indicating that the suturing method 

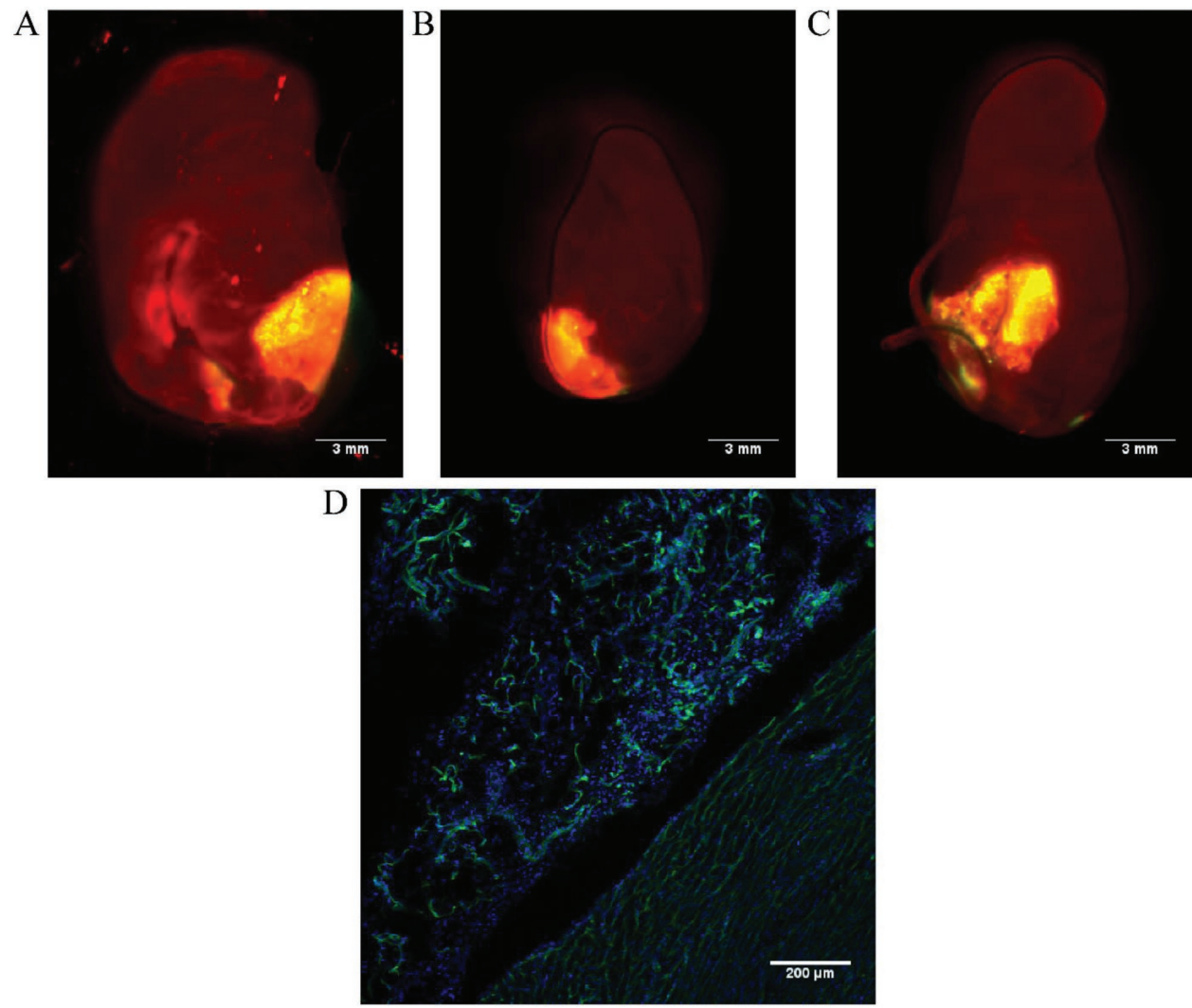

Figure 8. In vivo patch retention. hCPC-laden GelMA-cECM patches (yellow) are retained after $7 \mathrm{~d}$ : A) suture method and $14 \mathrm{~d}$; B) pericardial tucking method and C) suture method following implantation. D) Immunohistological analysis of vasculature formation (green) and cells (blue) after $14 \mathrm{~d}$ in vivo.

may be ideal for implementing a large device. In either case, the patches were retained throughout $14 \mathrm{~d}$ without change to patch shape, fluorescence expression of the cECM-bound dye, or buckling. The rat heart beats $\approx 400-500$ times $\mathrm{min}^{-1}$, significantly more than the human heart and thus these results are quite promising. Interactions between the patches and myocardium were evaluated using Isolectin staining for host vessel formation. As seen in Figure 8D, vessels were formed in the patches after $14 \mathrm{~d}$ in vivo, indicating that the patches integrate with the native myocardium and allow for nutrient delivery to the implanted cells. Overall, the patches were retained on the myocardium and became vascularized over the course of $14 \mathrm{~d}$. While this study only sought to determine the feasibility of use, future studies will examine the efficacy of the patch in diseased animal models in greater detail.

\section{Conclusions}

Here, we report the development of a novel pediatric hCPC/ cECM cardiac patch that was generated through bioprinting. The inclusion of $5 \% \mathrm{w} / \mathrm{v}$ GelMA allowed for printability of the hCPC/cECM bioink through GelMA polymerization via cooling to $10^{\circ} \mathrm{C}$, followed by white light radical polymerization and incubation at physiological temperatures. The inclusion of cECM allowed for improved printability over pure GelMA bioinks, and the hCPC-laden GelMA-cECM bioinks showed homogeneous distribution of cells and matrix. This methodology can potentially be employed to generate cardiac patches that can be customized to the target patient tissue. hCPCs remained highly viable and proliferative within the patch up to $7 \mathrm{~d}$ and hCPCs in GelMA-cECM patches had improved differentiation and angiogenic potential over pure GelMA patches, indicating their improved reparative functionality. The inclusion of cECM resulted in patches with a mechanical modulus that was similar to that of native myocardium and all patches were sufficiently hydrated. Patches did not significantly degrade over $21 \mathrm{~d}$ when tested in vitro through weight change and rheological analysis. In addition, hCPC-laden GelMA-cECM patches showed increased stiffness over $21 \mathrm{~d}$ when cultured in cFB conditioned media, indicating potential remodeling and retention in vivo. Finally, the printed GelMA-cECM patches were effectively attached to rat hearts epicardially, remained on the hearts for $14 \mathrm{~d}$, and showed vascularization.

The printing of native ECM is difficult to do at concentrations that can be realistically used and support cell functions. The concentration of cECM used here is similar to previous studies performed in our laboratory and by others that support CPC differentiation and function. In addition, all materials used in this study are clinically relevant as both cECM and hCPCs are in clinical testing alone. Thus, the idea of a patientspecific, 3D-printed patch is of great translational value. With printability achieved, future directions include incorporation of increased amounts of proregenerative ECM components such 
as agrin, sulfated glycosaminoglycans, or periostin that may improve the paracrine factor release by inducing modulations in hCPC function for a bottom-up, modular approach to tissue engineering. Additionally, evaluation of other critical bioactive factors such as cellular exosome release and the extent of attachment of the hCPCs within the patches may help evaluate how hCPCs are modified by inclusion of cECM and how the cellular changes effect paracrine release. Translation of the patch would involve the use to autologous hCPCs derived directly from patients, allowing for generation of patient-specific paracrine release while using commercially available biomaterials such as porcine cECM. Following hCPC expansion, patch manufacture can be expedited and customized through the 3D bioprinting methodology, which allows for incorporation of additional commercially available cell sources such as mesenchymal stem cells, and the tailored patch can be delivered directly to the patient for surgical attachment. Most importantly, therapeutic directions include testing the patches in vivo on animal models of RV failure to evaluate cellular changes such as angiogenesis, fibrosis, and cardiomyocyte proliferation, as well as tissue level parameters such as ejection fraction and RV wall thickness.

\section{Experimental Section}

Materials: GelMA was purchased from CellINK (Gothenburg, Sweden). Triethanolamine (TEOA), 1-vinyl-2-pyrrolidione (NVP), HEPES sodium salt, bovine gelatin, Tiron $\mathrm{X}-100$, sodium dodecyl sulfate, and pepsin were purchased from Sigma-Aldrich (MO, USA). Insulintransferrin-selenium (ITS) media, Hams F-12, Matrigel Matrix Growth Factor Reduced, Dulbecco's modified Eagle medium (DMEM), and penicillin-streptomycin (Pen-Strep) were purchased from Corning Cellgro (NY, USA). Eosin Y sodium salt was purchased from TCI (MA, USA). Nordson EFD 30cc barrels, pistons, and 27-gauge plastic tips were purchased from TEK products (MN, USA). Standard fetal bovine serum (FBS) and RNase-free water were purchased from Hyclone (PA, USA). L-glutamine was purchased from MP Biomedicals $(\mathrm{OH}, \mathrm{USA})$. Cell culture dishes and well plates were purchased from Cellstar (PA, USA). Calcein AM, ethidium homodimer-1 (EtD), DAPI, Vybrant DiD cell solution (DiD), Dynal magnetic beads, Trizol, Power SYBR Green, first strand buffer, dithiothreitol, Click-iT EdU Microplate Assay, RNaseOUT Inhibitor, M-MLV, Alexa Fluor 568 Carboxylic acid Succinimidyl Ester (AF568), and Alexa Fluor 790 Carboxylic acid Succinimidyl Ester (AF790) were purchased from Invitrogen (CA, USA). Phosphate buffered saline (PBS) and hexamers were purchased from Fisher Scientific $(\mathrm{NH}$, USA). Olido(dT) and dNTP were purchased from Fermentas (MA, USA). Primers were purchased from Integrated DNA Technologies (IL, USA). Fluorescein Griffonia Simplicifolia Lectin I Isolectin B4 (IsolectinB4-FITC) was purchased from Vector labs (CA, USA). HUVECs were purchased from Lonza (Basal, Switzerland). Endothelial cell growth media kits (include growth factors) were purchased from R\&D Systems (MN, USA). Hank's balanced salt solution (HBSS) was purchased from Gibco (OK, USA). Collagenase type $2\left(300 \mathrm{U} \mathrm{mL^{-1 } )}\right.$ was purchased from Worthington (NJ, USA). Anti-c-kit H300 antibodies were purchased from Santa Cruz (TX, USA). Bovine fibroblast growth factor (bFGF) was purchased from Stem Cell Technologies (Vancouver, Canada). QIAshredder centrifuge filters were purchased from QIAGEN (Hilden, Germany). Sprague-Dawley rats were purchased from Charles River (MA, USA). Isoflurane was purchased from Piramal Healthcare (Mumbai, India). Prolene was purchased from Ethicon (NJ, USA).

Neonatal Human Cardiac Progenitor Cell Isolation and Culture: The institutional review board at Children's Healthcare of Atlanta and Emory University approved the harvesting of human neonatal c-kit expressing hCPCs from atrial appendage, as previously described. ${ }^{[17,58]}$ In short, right atrial appendage tissue was obtained from pediatric patients aged one week or less undergoing heart surgeries due to congenital heart diseases. The atrial appendage tissue was transported using Krebs-Ringer solution, washed with HBSS, and broken down into small sections. The tissue was then enzymatically degraded using $1 \mathrm{mg} \mathrm{mL}^{-1}$ of collagenase type 11 at $37^{\circ} \mathrm{C}, 5 \% \mathrm{CO}_{2}$ for 30 min and passed through a $70 \mu \mathrm{m}$ filter. The mixture was centrifuged at $1000 \mathrm{~g}$ for $5 \mathrm{~min}$ to pellet the cells. The cells were combined with magnetic beads conjugated with anti-c-kit antibody, allowed to incubate for $2 \mathrm{~h}$ at $37^{\circ} \mathrm{C}$, followed by magnetic sorting and successive washes with cell culture media. Separated c-kit+ cells were expanded and expression of c-kit in the cell population was measured by flow cytometry to ensure they were at least $90 \%$ positive. Cells from three donors were pooled at first passage and used in all experiments described in this research. hCPCs were grown in $\mathrm{T}-75$ cell culture treated dishes with culture media for expansion. Media was changed every 2-3 d until bioink preparation. Cell culture media consisted of Ham's F-12 media supplemented with 1× Pen-Strep, 1\% L-glutamine, $10 \% \mathrm{FBS}$, and $10 \mathrm{ng} \mathrm{mL}^{-1} \mathrm{bFGF}$.

Cardiac Extracellular Matrix Isolation and Characterization: Decellularized porcine ventricular extracellular matrix (cECM) was generated and processed as previously described. ${ }^{[17,16,33]}$ Briefly, porcine ventricular tissue was separated, sectioned into small pieces, rinsed in PBS, and decellularized using a $1 \%$ solution of SDS for $4-5 \mathrm{~d}$. The decellularized $\mathrm{CECM}$ was rinsed with water, frozen at $-80^{\circ} \mathrm{C}$ overnight, lyophilized, and milled into a fine powder. Then, the $c E C M$ was processed into liquid form by partial digestion with pepsin $\left(1 \mathrm{mg} \mathrm{mL} \mathrm{L}^{-1}\right)$ in $0.1 \mathrm{M} \mathrm{HCl}$ for $2 \mathrm{~d}$, at a ratio of 10:1 of CECM to pepsin. The CECM was then raised to basic $\mathrm{pH}$ by adding $1 \mathrm{M} \mathrm{NaOH}$ and salt concentration of $1 \times$ PBS, followed by adjustment to $\mathrm{pH}$ of 7.4 using $\mathrm{HCl}$ and $\mathrm{NaOH}$ and diluted to a solution concentration of $8 \mathrm{mg} \mathrm{mL}^{-1}$. The solution was aliquoted, immediately frozen at $-80^{\circ} \mathrm{C}$ overnight, lyophilized for $24 \mathrm{~h}$, and stored at $-80^{\circ} \mathrm{C}$ prior to use.

Rat Cardiac Fibroblast Isolation and Conditioned Media Harvesting: cFBs were isolated as previously described. ${ }^{[59]}$ In short, hearts were excised from adult male rats, shredded, and subjected to digestion with $1 \mathrm{mg} \mathrm{mL} \mathrm{m}^{-1}$ trypsin in HBSS at $4{ }^{\circ} \mathrm{C}$ for $6 \mathrm{~h}$. The solutions were digested with $0.8 \mathrm{mg} \mathrm{mL}^{-1}$ collagenase in $\mathrm{HBSS}$ at $37^{\circ} \mathrm{C}$ for $15 \mathrm{~min}$ and then quenched with cell culture media. The cell suspensions were passed through $100 \mu \mathrm{m}$ filters, followed by cell pelleting and plating for $3 \mathrm{~h}$ to allow for cFB adherence, and then washed to remove non-cFBs. cFBs were grown in fibroblast growth media, which is composed of DMEM supplemented with $1 \times$ Pen-Strep, $1 \%$ L-glutamine, and 10\% FBS. cFB conditioned media was collected every $2 \mathrm{~d}$ from $\mathrm{CFBs}$ while they were grown until confluence, at which point the media across the entire culture time was combined and homogenized.

Bioink Preparation: GelMA solutions of $14.432 \%$ were created by dissolving lyophilized GelMA in $15 \times 10^{-3} \mathrm{M}$ HEPES buffer and allowed to dissolve under stirring at $60{ }^{\circ} \mathrm{C}$ for $1-2 \mathrm{~h}$. GelMA solutions were frozen at $-20{ }^{\circ} \mathrm{C}$ until use. Stock solutions of $13 \mathrm{mg} \mathrm{mL}^{-1} \mathrm{CECM}$ were formed by rehydrating the $\mathrm{CECM}$ material with HEPES buffer followed by adjusting the solution to $\mathrm{pH} 7.4$ with $\mathrm{HCl}$ immediately prior to use. All bioinks were prepared immediately before printing. GelMA bioinks were prepared by mixing GelMA (final concentration $5 \% \mathrm{w} / \mathrm{v}$ ), Eosin Y $\left(100 \times 10^{-6} \mathrm{M}\right)$, NVP $(0.75 \% \mathrm{v} / \mathrm{v})$, TEOA $(3 \% \mathrm{v} / \mathrm{v})$, and HEPES buffer $\left(15 \times 10^{-3} \mathrm{M}\right)$. In the case of GelMA-CECM bioinks, the HEPES in the above formulation was replaced by the CECM (final concentration $8 \mathrm{mg} \mathrm{mL} \mathrm{m}^{-1}$ ) solutions. For the inclusion of cells, neonatal hCPCs (passage 6-10) were removed from cell culture plates, pelleted at $1000 \mathrm{rpm}$ for $5 \mathrm{~min}$, and mixed with $1 \mathrm{~mL}$ solution of the bioink solution, producing a final concentration of 3 million cells $\mathrm{mL}^{-1}$.

Bioprinting and Patch Formation: All bioink solutions, with or without cells, underwent similar printing protocols. $1 \mathrm{~mL}$ of bioink was deposited into sterilized $30 \mathrm{cc}$ printer barrels and pushed toward the barrel head with a sterile loose fit plunger, removing any air bubbles that formed. A sterile 27-guage plastic needle tip was added to the barrel, and a cap connecting the print head to the barrel as added. The barrels were put in the low-temperature head of the bioprinter (EnvisionTEC 3D-bioplotter Developer Series), which was set to $10^{\circ} \mathrm{C}$, and the bioink 
was allowed to polymerize for $10 \mathrm{~min}$. After initial gelation, the printer head was calibrated and purged at 1.2 bar for $1-3 \mathrm{~s}$ to ensure free flowing and uniform filaments. Patches and grids were printed onto a glass slide platform at room temperature, using a pressure $0.7-0.8 \mathrm{bar}$ and speed of $10 \mathrm{~mm} \mathrm{~s}^{-1}$. Patches were $10 \mathrm{~mm}$ in diameter and $0.6 \mu \mathrm{m}$ thick, which printed in three layers, with an infill pattern of $90^{\circ}$ grids with $0.5 \mathrm{~mm}$ spacing. Six patches were printed at once. Test grids were $10 \mathrm{~mm} \times 10 \mathrm{~mm}$ boxes with an infill pattern of $90^{\circ}$ grids with $1 \mathrm{~mm}$ spacing. CAD models of the patches and grids printed were generated using SOLIDWORKS and imported to the printing control system through the Bioplotter RP program. Following printing, both patches and test grids were exposed to white light (Braintree Scientific) at $4{ }^{\circ} \mathrm{C}$ for $5 \mathrm{~min}$ to allow for radical polymerization. The patches were removed from glass slides and put in $2 \mathrm{~mL}$ solutions of cell culture media in 24-well plates. Patches that were being evaluated for paracrine release were put in $2 \mathrm{~mL}$ solutions of treatment media in 24-well plates. The treatment media consisted of Hams F-12 media supplemented with $1 \times$ Pen-Strep, $1 \%$ L-glutamine, $1 \times$ ITS, and $10 \mathrm{ng} \mathrm{mL}^{-1}$ bFGF. All patches were kept in cell culture incubators during further experiments, which allowed for CECM polymerization within $1 \mathrm{~h}$ after GelMA radical polymerization. Media was changed and/or harvested every $2 \mathrm{~d}$ for each group. The test grids followed a similar protocol after polymerization, with the difference being that the grids were not removed from the slides and media was added directly over the grids to allow for full and complete coverage. The test grids were left in the cell culture incubator for $1 \mathrm{~h}$ to allow for the CECM to fully polymerize, washed several times, and removed immediately for imaging.

Imaging Printed Test Grids: Imaging of the printed test grids was performed at 10× magnification with an Olympus $1 \times 71$ Inverted Microscope. Bright-field images of both GelMA and GelMA-cECM grids were taken for printability comparison. For evaluating $C E C M$ homogeneity throughout the printed structures, CECM solutions were allowed to bind with AF568 at $4{ }^{\circ} \mathrm{C}$ for $1 \mathrm{~h}$, which forms a strong bond to primary amines, at a concentration of $13.3 \mu \mathrm{g} \mathrm{mL}^{-1}$ based on modifying a previously described protocol. ${ }^{[60]}$ The $\mathrm{CECM}$ solution was used to form test grids as described in the printing section, followed by swelling and incubation for at least $1 \mathrm{~h}$ at $37^{\circ} \mathrm{C}$. Stained GelMA-CECM test grids were imaged at 10× magnification both on an Olympus $1 \times 71$ Inverted Microscope and Olympus FV1000 Confocal Microscope. Printed test grids with hCPCs were also imaged at 10x magnification with an Olympus $1 \times 71$ Inverted Microscope. To image only cells, hCPCs were incubated with the lyophilic dye DiD according to manufacture protocol. Briefly, hCPCs were trypsinized from cell culture dishes, counted, and pelleted. The cells were suspended at a density of 1 million cells $\mathrm{mL}^{-1}$ in serum-free culture media supplemented with $5 \mu \mathrm{L} \mathrm{mL}^{-1}$ of DiD solution (1 $\mathrm{mg} \mathrm{mL}^{-1}$ stock) and mixed. hCPCs were allowed to incubate for $20 \mathrm{~min}$ at $37^{\circ} \mathrm{C}$. The cells were centrifuged at $1000 \mathrm{rpm}$ for $5 \mathrm{~min}$, the supernatant was removed, and the cells were resuspended in serum-free media. The wash procedure was repeated twice to remove any unbound $\mathrm{DiD}$, and the cells were resuspended in the bioink solution for printing as described above. Printed test grids were again imaged at 10x on the fluorescence microscope. For image analysis of cell homogeneity throughout the printed structure, Imagel was used to measure several line scans of fluorescence intensity along grid lines, which were then averaged to produce the figure.

Printability Analysis: The printability analysis implemented in this work looks at the effectiveness of the extruded filaments in the test grids to form square holes between filaments, as previously described. ${ }^{[41]}$ Circularity $(C)$ of an enclosed area is based on the shape perimeter and area, where a perfect circle has a circularity of 1 . For a square shape, circularity is equal to $\pi / 4$. To this end, and as previously derived and defined, printability is given as Equation (1)

$\operatorname{Pr}=\frac{\pi}{4} \times \frac{1}{C}=\frac{L^{2}}{16 A}$

where $L$ is perimeter and $A$ is area of a shape. A printability of 1 is equal to a perfect square, and indicates optimal gelation, and thus printing, conditions of a bioink. Bright-field images of test grids were evaluated by measuring the perimeter and area of several holes in each sample and $\operatorname{Pr}$ was calculated using Equation (1), with three technical replicates and 4-6 holes per $n$.

Rheological Analysis: As the printed patches were too thick to be measured on a rheometer without rupture, disk-shaped hydrogels without cells were made by sandwiching $15 \mu \mathrm{L}$ of sample solution between two glass slides separated by a thin spacer, allowed to gel at $4{ }^{\circ} \mathrm{C}$ for $10 \mathrm{~min}$, and polymerized by white light (Braintree Scientific) for $5 \mathrm{~min}$ at $4{ }^{\circ} \mathrm{C}$. The sample disks were incubated overnight in cell treatment media to undergo CECM polymerization and swelling. The storage and loss moduli of the disks were measured using dynamic oscillatory strain and frequency sweeps performed on an Anton Paar MCR 302 stress-controlled rheometer with a $9 \mathrm{~mm}$ diameter $2^{\circ}$ measuring cone. ${ }^{[61]}$ The disks were loaded in the rheometer and the system was lowered to a $39 \mu \mathrm{m}$ gap. Strain amplitude sweeps were performed at $\omega=10 \mathrm{rad} \mathrm{s}^{-1}$ to determine the linear viscoelastic range of the samples. Oscillatory frequency sweeps between 0.5 and $30 \mathrm{rad} \mathrm{s}^{-1}$ and $2 \%$ strain were then used to measure the storage and loss moduli. Samples were measured at $1,4,7,12$, and $21 \mathrm{~d}$ for degradation analysis of cell-free samples grown in treatment media and at 1, 7, and $21 \mathrm{~d}$ for degradation analysis of cell-laden samples grown in cFB conditioned media. All samples had 3-6 technical replicates per $n$.

Swelling: Patches were printed without cells as described above and allowed to swell for $24 \mathrm{~h}$ in treatment media. The swollen patches were weighed (wet weight) and put in separate centrifuge tubes. The samples were lyophilized in a Labconvo lyophilizer for $2 \mathrm{~d}$ and the weight of the dried material was measured (dry weight). All samples had three technical replicates per $n$. Swelling ratio was calculated as wet weight/ dry weight.

Degradation: Patches were printed as described above and allowed to swell for at least $24 \mathrm{~h}$ in treatment media. For measuring of degradation via weight change, the patches were weighed at days $1,3,7,10,15$, and 21 after formation, and degradation via hydrolysis was determined as change in weight compared to original weight at day 1. For measurement of degradation via mechanics, as described in the Rheological Analysis section, mechanical measurements of the materials were taken at $1,4,7,12$, and $21 \mathrm{~d}$ for cell-free samples grown in treatment media, and at 1,7 , and $21 \mathrm{~d}$ for cell-laden samples grown in CFB conditioned media. Degradation was determined as the change in storage modulus (measured at $1.61 \mathrm{rad} \mathrm{s}^{-1}$ ) compared to original modulus at day 1. All samples had 3-6 technical replicates per $n$.

Viability Analysis: hCPC containing patches were grown in cell culture media for 1, 3, or $6 \mathrm{~d}$, changing media every $2 \mathrm{~d}$. Patches were removed from growth plates and placed in a $250 \mu \mathrm{L}$ solution of $3 \mu \mathrm{L} \mathrm{mL^{-1 }}$ Calcein AM (live) and $2 \mu \mathrm{L} \mathrm{mL} \mathrm{m}^{-1}$ EtD (dead) in HBSS in 48-well plates. The patches were left for $30 \mathrm{~min}$ at $37^{\circ} \mathrm{C}$ to incubate, followed by two washing with $1 \times$ HBSS for 5 min each. The patches were removed and placed on glass bottom dishes for imaging on an Olympus FV1000 Confocal Microscope. Live/dead images of the hCPCs within the patches were taken at several locations. Several areas of each patch, six patches each, were used as technical replicates to evaluate data expressed as live cells/total cells.

Tube Formation Assay: Conditioned media from empty or hCPC containing patches were grown in treatment media and collected at days 3 and 7. The conditioned media was centrifuged at $10000 \mathrm{~g}$ for $10 \mathrm{~min}$ to remove any cell debris or particulate matter, and the supernatant was stored at $-80^{\circ} \mathrm{C}$ until analysis. HUVECs were grown on $0.1 \% \mathrm{w} / \mathrm{v}$ gelatincoated T-75 tissue culture plates with endothelial cell growth media until assays were performed. Tube formation assays were implemented as previously described. ${ }^{[59,62]}$ In short, HUVECs were removed from culture using trypsin and added to Matrigel-coated well plates at a concentration of 10000 cells/well. Conditioned media harvested from patches $(200 \mu \mathrm{L})$ was added to the top of each well. For positive controls, nonconditioned treatment media was added. All HUVECs were allowed to grow for $6 \mathrm{~h}$. Calcein AM dye $\left(2 \mathrm{mg} \mathrm{mL}^{-1}\right)$ was added to each well and cells were imaged via fluorescence to measure tube formation. Extent of total tube length formed in each well was evaluated by the Angiogenesis Analyzer 
Table 1. PCR primers.

\begin{tabular}{|c|c|c|}
\hline Name & Forward 5'-3' & Reverse $5^{\prime}-3^{\prime}$ \\
\hline Connexin $43(\mathrm{Cx} 43)$ & $\begin{array}{c}\text { CAATCTCTCATCTCC- } \\
\text { GCTTCT }\end{array}$ & $\begin{array}{l}\text { GGCAACCTT- } \\
\text { GAGTTCTTCCTC }\end{array}$ \\
\hline GATA4 & $\begin{array}{l}\text { TAGACCGTCGGTTTTG- } \\
\text { CATTG }\end{array}$ & $\begin{array}{l}\text { CATCCACGTACATG- } \\
\text { GCAAACAG }\end{array}$ \\
\hline $\begin{array}{l}\text { Myocyte enhancement } \\
\text { factor 2C (MEF2C) }\end{array}$ & $\begin{array}{c}\text { TAACTTCTTTTCACTGTT- } \\
\text { GTGCTCCTT }\end{array}$ & GCCGCTTTTGGCAAATGTT \\
\hline $\begin{array}{l}\beta \text {-Myosin heavy chain } \\
\text { (MYH7) }\end{array}$ & $\begin{array}{l}\text { GGCAAGACAGTGACCGT- } \\
\text { GAAC }\end{array}$ & $\begin{array}{c}\text { CGTAGCGATCCTTGAG- } \\
\text { GTTGTA }\end{array}$ \\
\hline $\begin{array}{l}\text { Vascular endothelial } \\
\text { cadherin (VE-Cad) }\end{array}$ & $\begin{array}{l}\text { CCGACAGTTGTAG- } \\
\text { GCCCTGTT }\end{array}$ & $\begin{array}{l}\text { GGCATCTTCGGGTT- } \\
\text { GATCCT }\end{array}$ \\
\hline $\begin{array}{l}\text { Platelet endothelial cell } \\
\text { adhesion molecule (CD31) }\end{array}$ & $\begin{array}{c}\text { TCTATCACCTCGCCCTC- } \\
\text { CACAAA }\end{array}$ & $\begin{array}{l}\text { GAACGGTGTCTTCAG- } \\
\text { GTTCGTATTTCA }\end{array}$ \\
\hline $\begin{array}{l}\text { Vascular endothelial growth } \\
\text { factor receptor } 1 \text { (FLT-1) }\end{array}$ & $\begin{array}{l}\text { GACTAGATACCGTCAC- } \\
\text { CAGCAG }\end{array}$ & $\begin{array}{l}\text { GAAACCGT- } \\
\text { CACAATCCTCCTC }\end{array}$ \\
\hline $\begin{array}{l}\alpha \text {-Smooth muscle actin } \\
\text { (ACTA-2) }\end{array}$ & $\begin{array}{c}\text { AATACTCTGTCTGGATCG- } \\
\text { GTGGCT }\end{array}$ & $\begin{array}{c}\text { ACGAGTCAGAGCTTTG- } \\
\text { GCTAGGAA }\end{array}$ \\
\hline
\end{tabular}

for ImageJ (Gilles Carpentier), and the total tube length for each sample was normalized to the value of the positive controls.

RNA Isolation, Reverse Transcription, and Quantitative Real-Time $P C R$ : Cell-laden patches were grown in culture media for 3 and $7 \mathrm{~d}$. At each time point, three technical replicates $/ n$ were harvested, added to vials containing $1 \mathrm{~mL}$ Trizol to isolate RNA, and homogenized (Fisher Scientific PowerGen 500) for several minutes. ${ }^{[61]}$ The homogenized suspension was centrifuged at $15000 \mathrm{~g}$ for $1 \mathrm{~min}$ with QIAshredder filters to separate the cellular components from the gel. RNA extraction was performed according to manufacturer's protocols. RNA quantification and purity were determined by measuring absorbance at 260 and $280 \mathrm{~nm}$ wavelength on a spectrophotometer (Thermo Scientific NanoDrop One), followed by running reverse transcription as previously described. ${ }^{[16,17,61]}$ Briefly, 0.5-2 $\mu$ g RNA was mixed with hexamers, oligo(dT), dNTP, and RNase-free water in a final volume of $12 \mu \mathrm{L}$, and samples were heated to $65^{\circ} \mathrm{C}$ for 5 min to denature the RNA, followed by cooling to $25^{\circ} \mathrm{C}$ for $10 \mathrm{~min}$ to allow for components to anneal. Then, RNaseOUT inhibitor, $M-M L V$, first strand buffer, and dithiothreitol were added to solutions, heated to $37^{\circ} \mathrm{C}$ for $60 \mathrm{~min}$ to undergo reverse transcription, and $70^{\circ} \mathrm{C}$ for $15 \mathrm{~min}$ for enzyme inactivation. cDNA samples were stored at $-80^{\circ} \mathrm{C}$ prior to further measurement. Gene expression was measured using a quantitative real-time PCR system (Applied Biosystems, StepOne Plus Software). cDNA in 1:5 ratio was mixed with Power SYBER Green, RNase-free water, and target primer, heated to $95{ }^{\circ} \mathrm{C}$ for $10 \mathrm{~min}$, and allowed to run for 40 cycles, as previously described. ${ }^{[16,17]}$ Each sample was run in triplicate per primer, and $\Delta \Delta C_{t}$ method was used to obtain fold change values over GAPDH and GelMA control. ${ }^{[59]}$ The primer sequences used are seen in Table 1.

Proliferation: Cell-laden patches were grown in culture media supplemented with $20 \times 10^{-6} \mathrm{M}$ EdU for 3 and $7 \mathrm{~d}$. At each time point, patches were harvested and cut into equal size sections to fit in 96-well plates. Click-iT EdU assay was performed according to manufacturer's instructions. Briefly, cell-laden samples were fixed and incubated with Click-iT reaction cocktail. Samples were then incubated with anti-Oregon green HRP, followed by incubation in Amplex UltraRed reaction mixture. The reaction was stopped after $15 \mathrm{~min}$, absorbance of each well was measured, and absorbance of the blank was subtracted from each sample. Absorbance from GelMA-cECM patches was normalized to the absorbance of GelMA patches for each $n$.

Rat Surgery and Imaging: All animal experiments were performed with the approval of the Institutional Animal Care and Use Committee of Emory University. GelMA-CECM patches used for in vivo experiments were created in the same way as described in the Bioprinting section, with the difference being that AF568 dye was allowed to bind to $\mathrm{CECM}$ before patch formation, similar to the methodology described in the Imaging Printed Test Grids section. Sprague-Dawley Rats $(\approx 250 \mathrm{~g}$ in weight) were anesthetized with $2 \%$ isoflurane, intubated, and placed on the ventilator (Hallowell Emc Microvent 1). Following thoracotomy, the pericardial sac was then very carefully exposed and pulled back. The patch was then gently placed over the right ventricle of the heart, ensuring that there was no folding of the patch. Patches were left without further attachment, tucked underneath the pericardium, or attached the ventricle using a single suture. After 7 and $14 \mathrm{~d}$, rats were sacrificed and hearts were excised. Hearts were imaged using an Odyssey CLx (Li-Cor) for both patch fluorescence and heart background fluorescence, with an acquisition area of $20.07 \mathrm{~mm} \times 20.07 \mathrm{~mm}$.

Immunohistological Analysis: Excised hearts were fixed with 4\% PFA, sectioned, and mounted onto coverslips. Tissue sections were incubated overnight with IsolectinB4-FITC in blocking buffer (3\% BSA, 0.1\% Triton $\mathrm{X}-100,1 \times \mathrm{PBS})$ at $4{ }^{\circ} \mathrm{C}$. Sections were then incubated with DAPI and mounted. Tissue sections were imaged on an Olympus FV1000 Confocal Microscope.

Statistics: Numerical data are the mean \pm SEM. All data except for printability were analyzed using one-way ANOVAs with Tukey's multiple comparison post-test. Sample size ( $n$ ) was 3-6 for all samples. In cases where both days and groups were involved, data were compared across groups within the same day and across days within the same group to determine significant differences. An unpaired $t$-test was used to compare GelMA and GelMA-cECM in the printability analysis.

\section{Supporting Information}

Supporting Information is available from the Wiley Online Library or from the author.

\section{Acknowledgements}

This study was supported by the Betkowsi Family Research Fund to M.E.D. and the NHLBI (R01HL113468 to K.L.C.). D.B. was supported by the Cell and Tissue Engineering NIH Biotechnology Training Grant (T32 GM008433).

\section{Conflict of Interest}

K.L.C. is a cofounder, board member, consultant, and holds equity interest in Ventrix, Inc.

\section{Keywords}

bioprinting, cardiac extracellular matrix, cardiac patches, cardiac progenitor cells, pediatric heart failure

Received: June 11, 2018

Revised: October 16, 2018

Published online: October 31, 2018

[1] J. W. Rossano, J. J. Kim, J. A. Decker, J. F. Price, F. Zafar, D. E. Graves, D. L. Morales, J. S. Heinle, B. Bozkurt, S. W. Denfield, Circulation 2010, 122, A1374

[2] J. M. Robbins, T. M. Bird, J. M. Tilford, M. A. Cleves, C. A. Hobbs, S. D. Grosse, A. Correa, MMWR 2007, 56, 25.

[3] E. L. Bove, Pediatr. Cardiol. 1998, 19, 308.

[4] V. F. Segers, R. T. Lee, Nature 2008, 451, 937.

[5] R. Bolli, A. R. Chugh, D. D'Amario, J. H. Loughran, M. F. Stoddard, S. Ikram, G. M. Beache, S. G. Wagner, A. Leri, T. Hosoda, Lancet 2011, 378, 1847. 
[6] R. R. Makkar, R. R. Smith, K. Cheng, K. Malliaras, L. E. Thomson, D. Berman, L. S. Czer, L. Marbán, A. Mendizabal, P. V. Johnston, Lancet 2012, 379, 895.

[7] S. Ishigami, S. Ohtsuki, S. Tarui, D. Ousaka, T. Eitoku, M. Kondo, M. Okuyama, J. Kobayashi, K. Baba, S. Arai, Circ. Res. 2015, 116, 653.

[8] H. M. Burkhart, M. Y. Qureshi, S. C. Peral, P. W. O'leary, T. M. Olson, F. Cetta, T. J. Nelson, J. Thorac. Cardiovasc. Surg. 2015, 149, e35.

[9] U. Agarwal, A. W. Smith, K. M. French, A. V. Boopathy, A. George, D. Trac, M. E. Brown, M. Shen, R. Jiang, J. D. Fernandez, Stem Cells Transl. Med. 2016, 5, 883.

[10] L. Ye, W.-H. Zimmermann, D. J. Garry, J. Zhang, Circ. Res. 2013, $113,922$.

[11] D. A. Feyen, R. Gaetani, P. A. Doevendans, J. P. Sluijter, Adv. Drug Delivery Rev. 2016, 106, 104.

[12] E. Dawson, G. Mapili, K. Erickson, S. Taqvi, K. Roy, Adv. Drug Delivery Rev. 2008, 60, 215.

[13] M. Lutolf, J. Hubbell, Nat. Biotechnol. 2005, 23, 47.

[14] E. T. Pashuck, M. M. Stevens, Sci. Transl. Med. 2012, 4, 160 sr4.

[15] M. Gnecchi, Z. Zhang, A. Ni, V. J. Dzau, Circ. Res. 2008, 103, 1204.

[16] K. M. French, A. V. Boopathy, J. A. DeQuach, L. Chingozha, H. Lu, K. L. Christman, M. E. Davis, Acta Biomater. 2012, 8, 4357.

[17] K. M. French, J. T. Maxwell, S. Bhutani, S. Ghosh-Choudhary, M. J. Fierro, T. D. Johnson, K. L. Christman, W. R. Taylor, M. E. Davis, Stem Cell Transl. Med. 2016.

[18] J. M. Singelyn, J. A. DeQuach, S. B. Seif-Naraghi, R. B. Littlefield, P. J. Schup-Magoffin, K. L. Christman, Biomaterials 2009, 30, 5409.

[19] J. W. Wassenaar, R. Gaetani, J. J. Garcia, R. L. Braden, C. G. Luo, D. Huang, A. N. DeMaria, J. H. Omens, K. L. Christman, J. Am. Coll. Cardiol. 2016, 67, 1074.

[20] R. Gaetani, C. Yin, N. Srikumar, R. Braden, P. A. Doevendans, J. P. Sluijter, K. L. Christman, Cell Transplant. 2016, 25, 1653.

[21] G. Agmon, K. L. Christman, Curr. Opin. Solid State Mater. Sci. 2016, 20, 193.

[22] L. Yu, J. Ding, Chem. Soc. Rev. 2008, 37, 1473.

[23] B. Duan, Ann. Biomed. Eng. 2017, 45, 195.

[24] S. Kyle, Z. M. Jessop, A. Al-Sabah, I. S. Whitaker, Adv. Healthcare Mater. 2017, 6.

[25] S. V. Murphy, A. Skardal, A. Atala, J. Biomed. Mater. Res., Part A 2013, 101A, 272.

[26] F. Pati, J. Jang, D.-H. Ha, S. W. Kim, J.-W. Rhie, J.-H. Shim, D.-H. Kim, D.-W. Cho, Nat. Commun. 2014, 5, 3935.

[27] J. Jang, T. G. Kim, B. S. Kim, S.-W. Kim, S.-M. Kwon, D.-W. Cho, Acta Biomater. 2016, 33, 88.

[28] S. Huang, B. Yao, J. Xie, X. Fu, Acta Biomater. 2016, 32, 170.

[29] B. Xu, Y. Li, X. Fang, G. A. Thouas, W. D. Cook, D. F. Newgreen, Q. Chen, J. Mech. Behav. Biomed. Mater. 2013, 28, 354.

[30] Y. Efraim, H. Sarig, N. C. Anavy, U. Sarig, E. de Berardinis, S.-Y. Chaw, M. Krishnamoorthi, J. Kalifa, H. Bogireddi, T. V. Duc, Acta Biomater. 2017, 50, 220.

[31] S. Pok, O. M. Benavides, P. Hallal, J. G. Jacot, Tissue Eng., Part A 2014, 20, 1877.

[32] J. Jang, H.-J. Park, S.-W. Kim, H. Kim, J. Y. Park, S. J. Na, H. J. Kim, M. N. Park, S. H. Choi, S. H. Park, Biomaterials 2017, 112, 264.

[33] G. N. Grover, N. Rao, K. L. Christman, Nanotechnology 2014, 25, 014011.

[34] V. Hosseini, S. Ahadian, S. Ostrovidov, G. Camci-Unal, S. Chen, H. Kaji, M. Ramalingam, A. Khademhosseini, Tissue Eng., Part A 2012, 18, 2453

[35] T. Billiet, E. Gevaert, T. De Schryver, M. Cornelissen, P. Dubruel, Biomaterials 2014, 35, 49.
[36] G. Gao, A. F. Schilling, K. Hubbell, T. Yonezawa, D. Truong, Y. Hong, G. Dai, X. Cui, Biotechnol. Lett. 2015, 37, 2349.

[37] C. Bahney, T. Lujan, C. Hsu, M. Bottlang, J. West, B. Johnstone, Eur. Cells Mater. 2011, 22, 43.

[38] K. S. Lim, B. S. Schon, N. V. Mekhileri, G. C. Brown, C. M. Chia, S. Prabakar, G. J. Hooper, T. B. Woodfield, ACS Biomater. Sci. Eng. 2016, 2, 1752

[39] I. Noshadi, S. Hong, K. E. Sullivan, E. S. Sani, R. Portillo-Lara, A. Tamayol, S. R. Shin, A. E. Gao, W. L. Stoppel, L. D. Black III, A. Khademhosseini, N. Annabi, Biomater. Sci. 2017, 5, 2093.

[40] C. Colosi, S. R. Shin, V. Manoharan, S. Massa, M. Costantini, A. Barbetta, M. R. Dokmeci, M. Dentini, A. Khademhosseini, Adv. Mater. 2016, 28, 677.

[41] L. Ouyang, R. Yao, Y. Zhao, W. Sun, Biofabrication 2016, 8, 035020.

[42] K. Hölzl, S. Lin, L. Tytgat, S. Van Vlierberghe, L. Gu, A. Ovsianikov, Biofabrication 2016, 8, 032002.

[43] J. Li, M. Chen, X. Fan, H. Zhou, J. Transl. Med. 2016, 14, 271.

[44] R. Lev, D. Seliktar, J. R. Soc., Interface 2018, 15, 20170380.

[45] A. Blaeser, D. F. D. Campos, U. Puster, W. Richtering, M. M. Stevens, H. Fischer, Adv. Healthcare Mater. 2016, 5, 326.

[46] B. Liu, B. W. Lee, K. Nakanishi, A. Villasante, R. Williamson, J. Metz, J. Kim, M. Kanai, L. Bi, K. Brown, Nat. Biomed. Eng. 2018, 2, 293.

[47] A. Chierchia, N. Chirico, L. Boeri, I. Raimondi, G. A. Riva, M. T. Raimondi, M. Tunesi, C. Giordano, G. Forloni, D. Albani, Eur. J. Pharm. Biopharm. 2017, 121, 113.

[48] A. Skardal, S. V. Murphy, K. Crowell, D. Mack, A. Atala, S. Soker, J. Biomed. Mater. Res., Part B 2017, 105, 1986.

[49] A. Paul, A. Hasan, H. A. Kindi, A. K. Gaharwar, V. T. Rao, M. Nikkhah, S. R. Shin, D. Krafft, M. R. Dokmeci, D. Shum-Tim, ACS Nano 2014, 8, 8050.

[50] M. Stastna, J. E. Van Eyk, Circ. Genom. Precis. Med. 2012, 5, 8.

[51] S. B. Seif-Naraghi, D Horn, P. A. Schup-Magoffin, K. L. Christman, Acta Biomater. 2012, 8, 3695.

[52] S. Sonnenberg, A. A. Rane, C. J. Liu, N. Rao, G. Agmon, S. Suarez, R. Wang, A. Munoz, V. Bajaj, S. Zhang, R. Braden, P. J. Shup-Magoffin, O. L. Kwan, A. N. DeMaria, J. R. Cochran, K. L. Christman, Biomaterials 2015, 45, 56.

[53] B. Trappmann, J. E. Gautrot, J. T. Connelly, D. G. Strange, Y. Li, M. L. Oyen, M. A. C. Stuart, H. Boehm, B. Li, V. Vogel, Nat. Mater. 2012, 11, 642

[54] C. Williams, E. Budina, W. L. Stoppel, K. E. Sullivan, S. Emani, S. M. Emani, L. D. Black III, Acta Biomater. 2015, 14, 84.

[55] J. K. Nguyen, D. J. Park, J. L. Skousen, A. E. Hess-Dunning, D. J. Tyler, S. J. Rowan, C. Weder, J. R. Capadona, J. Neural Eng. 2014, 11, 056014.

[56] J. W. Nichol, S. T. Koshy, H. Bae, C. M. Hwang, S. Yamanlar, A. Khademhosseini, Biomaterials 2010, 31, 5536.

[57] V. V. Patel, L. Zhao, P. Wong, B. B. Pradhan, H. W. Bae, L. Kanim, R. B. Delamarter, Spine J. 2006, 6, 397.

[58] U. Agarwal, A. George, S. Bhutani, S. Ghosh-Choudhary, J. T. Maxwell, M. E. Brown, Y. Mehta, M. O. Platt, Y. Liang, S. Sahoo, M. E. Davis, Circ. Res. 2017, 120, 701.

[59] W. D. Gray, K. M. French, S. Ghosh-Choudhary, J. T. Maxwell, M. E. Brown, M. O. Platt, C. D. Searles, M. E. Davis, Circ. Res. 2015, 116, 255.

[60] J. W. Wassenaar, R. L. Braden, K. G. Osborn, K. L. Christman, J. Mater. Chem. B 2016, 4, 2794.

[61] S. Bhutani, A. L. Nachlas, M. E. Brown, T. Pete, C. T. Johnson, A. J. García, M. E. Davis, ACS Biomater. Sci. Eng. 2018, 4, 200.

[62] K. L. DeCicco-Skinner, G. H. Henry, C. Cataisson, T. Tabib, J. C. Gwilliam, N. J. Watson, E. M. Bullwinkle, L. Falkenburg, R. C. O'Neill, A. Morin, J. S. Wiest, J. Visualized Exp. 2014, e51312. 